\title{
Preparation of $\mathrm{BiFeO}_{3}$-Graphene Nanocomposites and Their Enhanced Photocatalytic Activities
}

\author{
J. F. Dai, ${ }^{1,2}$ T. Xian, ${ }^{1,2}$ L. J. Di, ${ }^{2}$ and H. Yang ${ }^{1,2}$ \\ ${ }^{1}$ State Key Laboratory of Gansu Advanced Non-Ferrous Metal Materials, Lanzhou University of Technology, Lanzhou 730050, China \\ ${ }^{2}$ School of Science, Lanzhou University of Technology, Lanzhou 730050, China \\ Correspondence should be addressed to H. Yang; hyang@lut.cn
}

Received 23 September 2013; Accepted 27 October 2013

Academic Editor: Jian Lin

Copyright (c) 2013 J. F. Dai et al. This is an open access article distributed under the Creative Commons Attribution License, which permits unrestricted use, distribution, and reproduction in any medium, provided the original work is properly cited.

\begin{abstract}
$\mathrm{BiFeO}_{3}$ nanoparticles were prepared via a polyacrylamide gel route. $\mathrm{BiFeO}_{3}$-graphene nanocomposites were fabricated by mixing $\mathrm{BiFeO}_{3}$ nanoparticles and graphene into absolute ethanol solution followed by thermal drying. The TEM observation demonstrates that the $\mathrm{BiFeO}_{3}$ nanoparticles are well anchored onto graphene sheets. The photocatalytic activities of the as-prepared samples were evaluated by the degradation of methyl orange $(\mathrm{MO})$ under simulated sunlight irradiation. Compared to bare $\mathrm{BiFeO}_{3}$ nanoparticles, $\mathrm{BiFeO}_{3}$-graphene nanocomposites exhibit enhanced photocatalytic activity. The outstanding photocatalytic performance is mainly ascribed to the efficient transfer of photogenerated electrons from $\mathrm{BiFeO}_{3}$ to graphene, thus leading to an increased availability of $\mathrm{h}^{+}$for the photocatalytic reaction. In addition, hydroxyl $(\cdot \mathrm{OH})$ radicals were detected by the photoluminescence technique using terephthalic acid as a probe molecule and are found to be produced on the irradiated $\mathrm{BiFeO}_{3}$ and $\mathrm{BiFeO}_{3}$-graphene nanocomposites; in particular, an enhanced yield is observed for the latter.
\end{abstract}

\section{Introduction}

Semiconductor-based photocatalysts have attracted considerable attention over the past decades due to their potential applications in solar energy conversion and environmental purification $[1,2]$. Among them, $\mathrm{TiO}_{2}$ has proven to be a powerful photocatalyst for the degradation of numerous organic compounds [3-5]. However, it can only respond to UV light owing to its wide bandgap $(\sim 3.2 \mathrm{eV})$. To efficiently make use of solar energy that consists largely of visible light, it is essential to explore visible-light-driven photocatalysts.

$\mathrm{BiFeO}_{3}$, exhibiting simultaneous ferroelectric and antiferromagnetic properties at room temperature, has been extensively studied as one of the most important multiferroic materials $[6,7]$. Besides its multiferroic property, recent investigations have revealed that $\mathrm{BiFeO}_{3}$ also exhibits visiblelight responsive photocatalytic activity for the degradation of organic pollutants [8-13]. Generally, the overall photocatalytic activity of a photocatalyst depends on numerous factors. Among them, the effective separation of photogenerated electron-hole $\left(\mathrm{e}^{-}-\mathrm{h}^{+}\right)$pairs is very important in improving the photocatalytic activity. Graphene, being a two-dimensional (2D) sheet of $s p^{2}$-hybridized carbon atoms, possesses excellent properties including high electrical conductivity, electron mobility, thermal conductivity, mechanical strength, chemical stability, and so forth [14-16]. On account of its outstanding properties, graphene has been frequently used as an ideal support to integrate with a large number of functional nanomaterials to form unique nanocomposites with improved performances in the fields of photocatalysts [17-23], microsupercapacitors [24], field-emission emitters [25], and fuel cells [26]. Particularly, the combination of graphene with photocatalysts is demonstrated to be an efficient way to promote the separation of photogenerated $\mathrm{e}^{-}$$\mathrm{h}^{+}$pairs and then enhance their photocatalytic activities [1723]. In these graphene-photocatalyst composites, photogenerated electrons can be readily captured by graphene which acts as electron acceptor; thus, more photogenerated holes are increasingly available for the photocatalytic reactions. Therefore, many efforts have been devoted to incorporating graphene into $\mathrm{BiFeO}_{3}$-based composite materials.

Up to now, the $\mathrm{BiFeO}_{3}$-graphene composites are often prepared by hydrothermal method and sol-gel route. In 


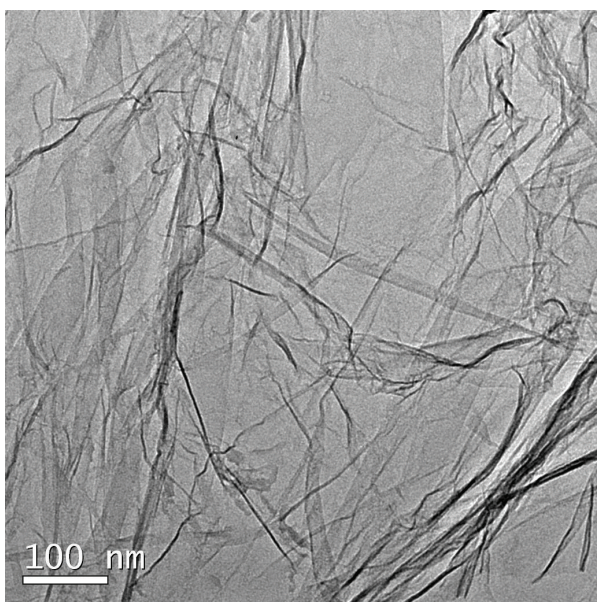

(a)

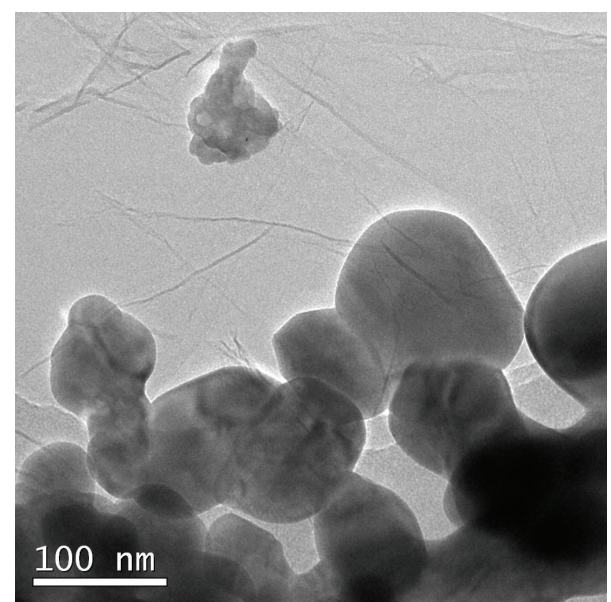

(b)

Figure 1: TEM images of graphene and $\mathrm{BiFeO}_{3}$-graphene(5\%) nanocomposite.

the former method, the $\mathrm{BiFeO}_{3}$ precursors were introduced into the dispersed aqueous of graphene or graphene oxide, followed by hydrothermal reaction, to yield $\mathrm{BiFeO}_{3}$-graphene composites [23, 27]. For the latter route, the sol containing precursors and graphene oxide was dried, and then the resultant was thermally treated at $\mathrm{N}_{2}$ atmosphere, leading to the formation of $\mathrm{BiFeO}_{3}$-graphene hybrids [22]. However, it should be noted that the aforementioned processes are based on hydrothermal condition or high temperature thermal treatment procedure, which may damage the graphene lattice [28]. In this paper, we demonstrate that $\mathrm{BiFeO}_{3}$ nanoparticles can be assembled onto graphene sheets by mixing $\mathrm{BiFeO}_{3}$ nanoparticles and graphene into absolute ethanol solution followed by thermal drying. The photocatalytic activities of prepared samples were evaluated by the degradation of methylene orange (MO) under simulated sunlight irradiation. The production of ${ }^{\bullet} \mathrm{OH}$ radicals on the irradiated $\mathrm{BiFeO}_{3}$-graphene photocatalyst was examined by the photoluminescence (PL) technique using terephthalic acid (TPA) as a probe molecule.

\section{Experimental}

The graphene used in this research was purchased from Nanjing XFNano Materials Tech Co. Ltd. $\mathrm{BiFeO}_{3}$ nanoparticles were synthesized via a polyacrylamide gel route as described in the literature [29]. To assemble $\mathrm{BiFeO}_{3}$ nanoparticles on graphene, number of $\mathrm{BiFeO}_{3}$ nanoparticles and graphene were dispersed into absolute ethanol solution and ultrasonically treated for $10 \mathrm{~min}$. The obtained mixture was dried at $60^{\circ} \mathrm{C}$ for $10 \mathrm{~h}$ in a thermostat drier, during which ethanol was vaporized, leaving behind $\mathrm{BiFeO}_{3}$ nanoparticles well anchored on graphene nanosheets. By changing the graphene content, several $\mathrm{BiFeO}_{3}$-graphene nanocomposite samples with graphene weight fractions of $1 \%, 3 \%, 5 \%, 7 \%$, and $9 \%$ were prepared.

The photocatalytic activity of the $\mathrm{BiFeO}_{3}$-graphene nanocomposites was evaluated by the degradation of $\mathrm{MO}$ under simulated sunlight irradiation from a $200 \mathrm{~W}$ xenon lamp (incident light power: $\sim 5 \mathrm{~mW} \mathrm{~cm}^{-2}$ ) at room temperature. The initial dye concentration was $10 \mathrm{mg} \mathrm{L}^{-1}$ with a catalyst loading of $2.5 \mathrm{~g} \mathrm{~L}^{-1}$. Before illumination, the mixed solution was mildly stirred for $1 \mathrm{~h}$ in the dark in order to reach the adsorption-desorption equilibrium of $\mathrm{MO}$ on the catalyst. The concentration of MO after photocatalytic degradation was determined by measuring the absorbance of the solution at a fixed wavelength of $464 \mathrm{~nm}$ using a UV-visible spectrophotometer. Before the absorbance measurements, the reaction solution was centrifuged for $10 \mathrm{~min}$ at $4000 \mathrm{r} \mathrm{min}^{-1}$ to remove the catalyst.

Terephthalic acid (TPA) was used as a probe molecule to examine ${ }^{\circ} \mathrm{OH}$ radicals formed on the irradiated $\mathrm{BiFeO}_{3}$ graphene photocatalyst. It is expected that TPA reacts with ${ }^{\circ} \mathrm{OH}$ to generate a highly fluorescent compound, 2hydroxyterephthalic acid (TAOH). By measuring the PL intensity of $\mathrm{TAOH}$ that is pronounced around $429 \mathrm{~nm}$, the information about ${ }^{\circ} \mathrm{OH}$ can be obtained. TPA was dissolved in $1.0 \times 10^{-3} \mathrm{~mol} \mathrm{~L}^{-1} \mathrm{NaOH}$ aqueous solution to make a $2.5 \times 10^{-4} \mathrm{~mol} \mathrm{~L}^{-1} \mathrm{TPA}$ solution, and then to the solution was added $2.5 \mathrm{~g} \mathrm{~L}^{-1}$ photocatalyst. The mixed solution, after several minutes of ultrasound treatment in the dark, was illuminated under the $200 \mathrm{~W}$ xenon lamp. The reacted solution was centrifuged for $10 \mathrm{~min}$ at $4000 \mathrm{rmin}^{-1}$ to separate the catalyst particles and was then used for the PL measurements at a fluorescence spectrophotometer with the excitation wavelength of $315 \mathrm{~nm}$.

The morphology of the sample was observed using a field-emission transmission electron microscope (TEM). A fluorescence spectrophotometer was used to measure the photoluminescence (PL) emission spectra of the samples.

\section{Results and Discussion}

Figure 1(a) presents the TEM image of pure graphene, indicating that graphene has typical two-dimensional sheet structure with crumpled feature. Figure 1(b) shows the TEM image of $\mathrm{BiFeO}_{3}$-graphene $(5 \%)$ nanocomposite. It can be seen that $\mathrm{BiFeO}_{3}$ nanoparticles are assembled onto the graphene sheet. 


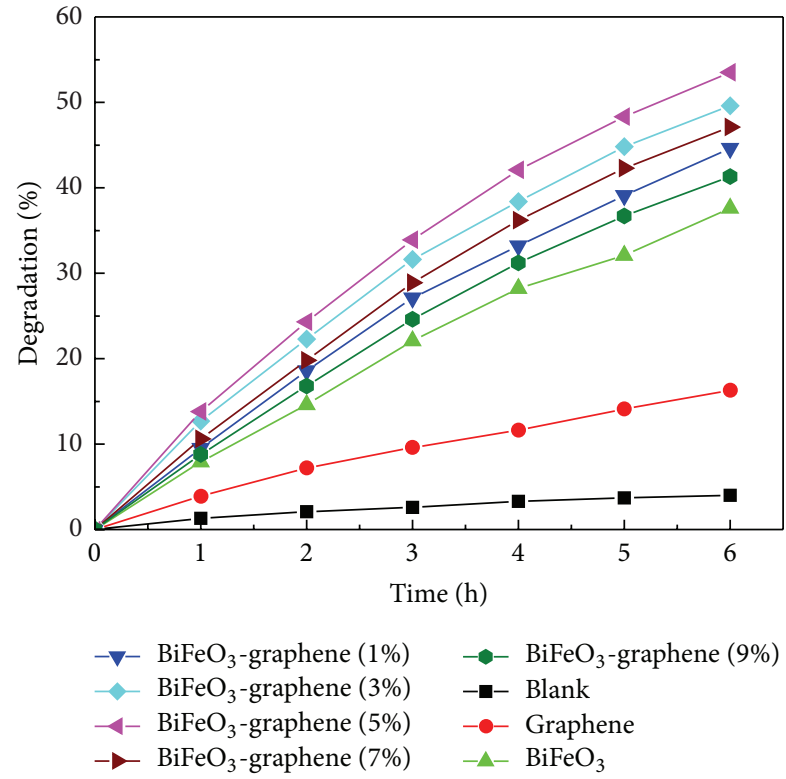

FIgure 2: Photocatalytic degradation of $\mathrm{MO}$ as a function of irradiation time over bare $\mathrm{BiFeO}_{3}$ nanoparticles and graphene and $\mathrm{BiFeO}_{3}$ graphene nanocomposites with graphene weight fractions of $1 \%, 3 \%$, $5 \%, 7 \%$, and $9 \%$.

The $\mathrm{BiFeO}_{3}$ nanoparticles mainly exhibit sphere-like shape and have an average particle size centered around $110 \mathrm{~nm}$.

Figure 2 shows the photocatalytic degradation of MO over $\mathrm{BiFeO}_{3}$-graphene nanocomposites as a function of irradiation time $(t)$. The degradation percentage is defined as $\left(C_{0}-C_{t}\right) / C_{0} \times 100 \%$, where $C_{0}$ and $C_{t}$ are the MO concentrations before and after irradiation, respectively. The blank experiment result is also shown in Figure 2, from which one can see that the MO is hardly degraded under simulated sunlight irradiation without photocatalysts, and its degradation percentage is less than $5 \%$ after $6 \mathrm{~h}$ of exposure. After $6 \mathrm{~h}$ irradiation in the presence of $\mathrm{BiFeO}_{3}$ nanoparticles and graphene, about $37 \%$ and $16 \%$ of $\mathrm{MO}$ are observed to be degraded, respectively. When $\mathrm{BiFeO}_{3}$ nanoparticles assembled on graphene, all samples of $\mathrm{BiFeO}_{3}$-graphene nanocomposites exhibit higher photocatalytic activity than bare $\mathrm{BiFeO}_{3}$ nanoparticles. Moreover, the photocatalytic activity of nanocomposites increases gradually with the increase in graphene content from $1 \%$ to $5 \%$. Further increase in graphene content leads to the decrease of photocatalytic activity.

Figure 3 shows the PL spectra of the TPA solution after reacting $6 \mathrm{~h}$ over the simulated sunlight irradiated $\mathrm{BiFeO}_{3}$ and $\mathrm{BiFeO}_{3}$-graphene $(5 \%)$ photocatalysts. The blank experiment result indicates that no PL signal is observed at $429 \mathrm{~nm}$ after irradiation without catalyst. With $\mathrm{BiFeO}_{3}$ as photocatalyst, the PL signal centered around $429 \mathrm{~nm}$ is detected, revealing that ${ }^{\circ} \mathrm{OH}$ radicals are generated on the irradiated $\mathrm{BiFeO}_{3}$. When $\mathrm{BiFeO}_{3}$-graphene $(5 \%)$ nanocomposite is used as the photocatalyst, the PL signal intensity is slightly increased, which suggests that the yield of ${ }^{\circ} \mathrm{OH}$ radicals is enhanced on the irradiated nanocomposite.

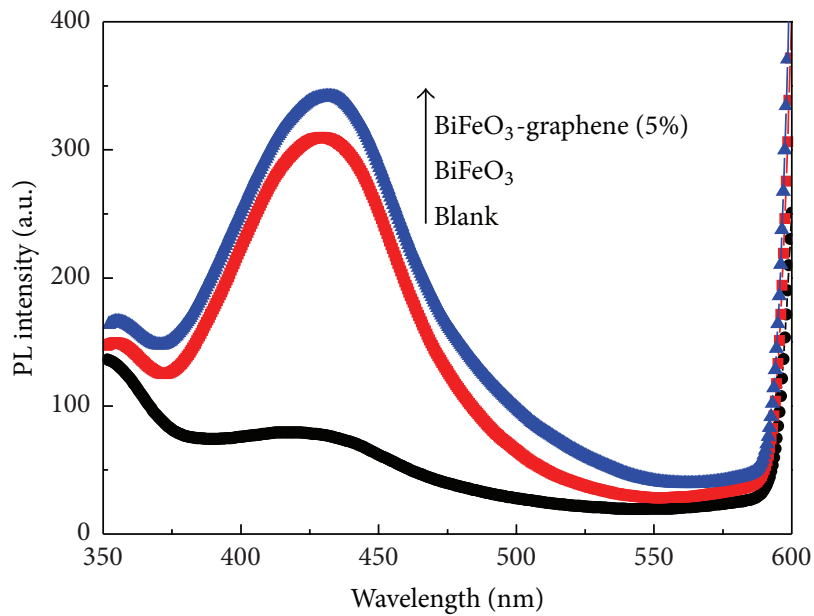

FIGURE 3: PL spectra of the TPA solution after reacting $6 \mathrm{~h}$ over the irradiated $\mathrm{BiFeO}_{3}$ nanoparticles and $\mathrm{BiFeO}_{3}$-graphene (5\%) nanocomposite.

Figure 4 schematically shows the photocatalytic mechanism of $\mathrm{BiFeO}_{3}$-graphene nanocomposite toward the degradation of MO. Under the simulated sunlight irradiation, the valence band $(\mathrm{VB})$ electrons of $\mathrm{BiFeO}_{3}$ are promoted to the conduction band $(\mathrm{CB})$, inducing the production of $\mathrm{e}^{-}-\mathrm{h}^{+}$pairs. The photogenerated electrons and holes then participate in a series of redox reactions to form a number of active species. However, the redox reaction processes are strongly related to the $\mathrm{CB}$ and $\mathrm{VB}$ edge potentials of $\mathrm{BiFeO}_{3}$. The VB potential of the $\mathrm{BiFeO}_{3}$ can be calculated using the following relation $[30]$ :

$$
E_{\mathrm{VB}}=X-E^{\mathrm{e}}+0.5 E_{\mathrm{g}}
$$

where $X$ is the absolute electronegativity of the semiconductor, $E_{\mathrm{e}}$ is the energy of free electrons on the hydrogen scale $(\sim 4.5 \mathrm{eV})$, and $E_{\mathrm{g}}$ is the bandgap energy of the semiconductor (for the as-prepared $\mathrm{BiFeO}_{3}, E_{\mathrm{g}}$ is $2.06 \mathrm{eV}$ [31]). The value of $X$ for $\mathrm{BiFeO}_{3}$ is obtained, by the arithmetic mean of the electron affinity and the first ionization of the constituent atoms reported in the literatures $[32,33]$, to be $5.93 \mathrm{eV}$. Thus, the $\mathrm{CB}$ and $\mathrm{VB}$ potentials of $\mathrm{BiFeO}_{3}$ are calculated to be 0.4 and $2.46 \mathrm{~V}$ versus normal hydrogen electrode (NHE), respectively. It can be seen that the VB potential of sample is more positive than the redox potential of $\mathrm{OH}^{-} /{ }^{\circ} \mathrm{OH}$ $(1.89 \mathrm{~V} / \mathrm{NHE})$, indicating that the photogenerated holes have strong oxidative ability and they can oxide $\mathrm{OH}^{-}$into ${ }^{\circ} \mathrm{OH}$. However, the $\mathrm{CB}$ potential of sample is not negative enough to reduce $\mathrm{O}_{2}$ to $\mathrm{O}_{2}{ }^{-}{ }^{-}(-0.13 \mathrm{~V} / \mathrm{NHE})$ via $\mathrm{e}^{-}$. As a result, it is reasonable to infer that ${ }^{\circ} \mathrm{OH}$ radicals derived by the reaction of the photogenerated $\mathrm{h}^{+}$with $\mathrm{OH}^{-}$are the main active species responsible for the degradation of $\mathrm{MO}$ over simulated sunlight irradiated $\mathrm{BiFeO}_{3}$. Therefore, the effective separation of $\mathrm{e}^{-}-\mathrm{h}^{+}$pairs and increased availability of $\mathrm{h}^{+}$are the key points to improve the photocatalytic activity of the $\mathrm{BiFeO}_{3}$. When $\mathrm{BiFeO}_{3}$ nanoparticles assembled on grapheme, that is, an excellent electron acceptor and conductor, the photogenerated electrons readily transfer from the $\mathrm{BiFeO}_{3}$ conduction 


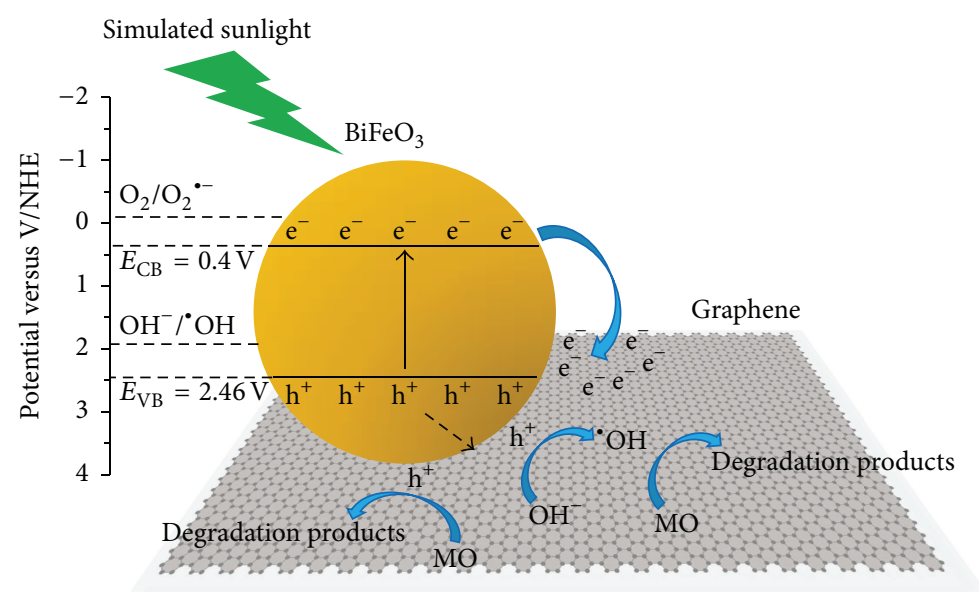

FIGURE 4: Schematic illustration of the photocatalytic mechanism of $\mathrm{BiFeO}_{3}$-graphene nanocomposite toward the degradation of MO.

band to graphene, which could suppress the recombination of photoexcited $\mathrm{e}^{-}-\mathrm{h}^{+}$pairs, thus leading to the increase in the number of holes which participate in the photocatalytic reaction. The results shown in Figure 3 confirm the enhanced yield of ${ }^{\circ} \mathrm{OH}$ radicals on the irradiated $\mathrm{BiFeO}_{3}$ graphene nanocomposite. As a result, with the introduction of an amount of graphene, the resulted $\mathrm{BiFeO}_{3}$-graphene nanocomposites exhibit an improved photocatalytic activity compared to bare $\mathrm{BiFeO}_{3}$ nanoparticles. However, when the graphene content is further increased above its optimum value, the photocatalytic efficiency begins to exhibit a decreasing trend. This is ascribed to the following reasons: (i) the excessive graphene may shield the light and decrease the number of photon absorption on $\mathrm{BiFeO}_{3}$ particles; (ii) the amount of available surface active sites tends to be reduced due to an increasing coverage of graphene onto the surface of $\mathrm{BiFeO}_{3}$ particles.

\section{Conclusions}

$\mathrm{BiFeO}_{3}$ nanoparticles were prepared via a polyacrylamide gel method. $\mathrm{BiFeO}_{3}$-graphene nanocomposites were fabricated by mixing $\mathrm{BiFeO}_{3}$ nanoparticles and graphene into ethanol followed by thermal drying at $60^{\circ} \mathrm{C}$. It is found that the $\mathrm{BiFeO}_{3}$ nanoparticles are well anchored onto graphene sheet. The photocatalytic experiments indicate that the $\mathrm{BiFeO}_{3}$-graphene nanocomposites exhibit higher photocatalytic activity for the degradation of $\mathrm{MO}$ under simulated sunlight irradiation than bare $\mathrm{BiFeO}_{3}$ nanoparticles, which is attributed to the fact that the photogenerated electrons are captured by graphene, leading to an increased availability of $\mathrm{h}^{+}$for the photocatalytic reaction.

\section{Acknowledgments}

This work was supported by the National Natural Science Foundation of China (Grant nos. 50962009 and 51262018) and the Hongliu Outstanding Talents Foundation of Lanzhou University of Technology (Grant no. J201205).

\section{References}

[1] A. Mills, R. H. Davies, and D. Worsley, "Water purification by semiconductor photocatalysis," Chemical Society Reviews, vol. 22, no. 6, pp. 417-425, 1993.

[2] M. R. Hoffmann, S. T. Martin, W. Choi, and D. W. Bahnemann, "Environmental applications of semiconductor photocatalysis," Chemical Reviews, vol. 95, no. 1, pp. 69-96, 1995.

[3] J. H. Carey, J. Lawrence, and H. M. Tosine, "Photodechlorination of PCB's in the presence of titanium dioxide in aqueous suspensions," Bulletin of Environmental Contamination and Toxicology, vol. 16, no. 6, pp. 697-701, 1976.

[4] S. Mozia, P. Brozek, J. Przepiorski, B. Tryba, and A. W. Morawki, "Immobilized $\mathrm{TiO}_{2}$ for phenol degradation in a pilot-scale photocatalytic reactor," Journal of Nanomaterials, vol. 2012, Article ID 949764, p. 10, 2012.

[5] Y. Han, H.-S. Kim, and H. Kim, "Relationship between synthesis conditions and photocatalytic activity of nanocrystalline $\mathrm{TiO}_{2}$," Journal of Nanomaterials, vol. 2012, Article ID 427453, 10 pages, 2012.

[6] J. Wang, J. B. Neaton, H. Zheng et al., "Epitaxial $\mathrm{BiFeO}_{3}$ multiferroic thin film heterostructures," Science, vol. 299, no. 5613, pp. 1719-1722, 2003.

[7] G. Catalan and J. F. Scott, "Physics and applications of bismuth ferrite," Advanced Materials, vol. 21, no. 24, pp. 2463-2485, 2009.

[8] F. Gao, X. Chen, K. Yin et al., "Visible-light photocatalytic properties of weak magnetic $\mathrm{BiFeO}_{3}$ nanoparticles," Advanced Materials, vol. 19, no. 19, pp. 2889-2892, 2007.

[9] R. Guo, L. Fang, W. Dong, F. Zheng, and M. Shen, "Magnetically separable $\mathrm{BiFeO}_{3}$ nanoparticles with a $\gamma-\mathrm{Fe}_{2} \mathrm{O}_{3}$ parasitic phase: controlled fabrication and enhanced visible-light photocatalytic activity," Journal of Materials Chemistry, vol. 21, no. 46, pp. 18645-18652, 2011.

[10] S. Li, Y.-H. Lin, B.-P. Zhang, Y. Wang, and C.-W. Nan, "Controlled fabrication of $\mathrm{BiFeO}_{3}$ uniform microcrystals and their magnetic and photocatalytic behaviors," Journal of Physical Chemistry C, vol. 114, no. 7, pp. 2903-2908, 2010.

[11] J. Wei, C. Zhang, and Z. Xu, "Low-temperature hydrothermal synthesis of $\mathrm{BiFeO}_{3}$ microcrystals and their visible-light photocatalytic activity," Materials Research Bulletin, vol. 47, no. 11, pp. 3513-3517, 2012. 
[12] Y. Huo, Y. Jin, and Y. Zhang, "Citric acid assisted solvothermal synthesis of $\mathrm{BiFeO}_{3}$ microspheres with high visible-light photocatalytic activity," Journal of Molecular Catalysis A, vol. 331, no. 1-2, pp. 15-20, 2010.

[13] C. Reitz, C. Suchomski, C. Weidmann, and T. Brezesinski, "Block copolymer-templated $\mathrm{BiFeO}_{3}$ nanoarchitectures composed of phase-pure crystallites intermingled with a continuous mesoporosity: effective visible-light photocatalysts?" Nano Research, vol. 4, no. 4, pp. 414-424, 2011.

[14] K. I. Bolotin, K. J. Sikes, Z. Jiang et al., "Ultrahigh electron mobility in suspended graphene," Solid State Communications, vol. 146, no. 9-10, pp. 351-355, 2008.

[15] A. A. Balandin, S. Ghosh, W. Bao et al., "Superior thermal conductivity of single-layer graphene," Nano Letters, vol. 8, no. 3, pp. 902-907, 2008.

[16] I. W. Frank, D. M. Tanenbaum, A. M. Van Der Zande, and P. L. McEuen, "Mechanical properties of suspended graphene sheets," Journal of Vacuum Science and Technology B, vol. 25, no. 6, pp. 2558-2561, 2007.

[17] X. Liu, L. Pan, Q. Zhao et al., "UV-assisted photocatalytic synthesis of $\mathrm{ZnO}$-reduced graphene oxide composites with enhanced photocatalytic activity in reduction of $\mathrm{Cr}(\mathrm{VI})$," Chemical Engineering Journal, vol. 183, pp. 238-243, 2012.

[18] H. Fan, X. Zhao, J. Yang et al., "ZnO-graphene composite for photocatalytic degradation of methylene blue dye," Cataylsis Communication, vol. 29, pp. 29-34, 2012.

[19] S. Liu, J. Tian, L. Wang, Y. Luo, and X. Sun, "One-pot synthesis of $\mathrm{CuO}$ nanoflower-decorated reduced graphene oxide and its application to photocatalytic degradation of dyes," Catalysis Science and Technology, vol. 2, no. 2, pp. 339-344, 2012.

[20] S. Song, W. Gao, X. Wang et al., "Microwave-assisted synthesis of $\mathrm{BiOBr} /$ graphene nanocomposites and their enhanced photocatalytic activity," Dalton Transactions, vol. 41, no. 34, pp. 1047210476, 2012.

[21] G. Chen, M. Sun, Q. Wei, Y. Zhang, B. Zhu, and B. Du, " $\mathrm{Ag}_{3} \mathrm{PO}_{4}$ /graphene-oxide composite with remarkably enhanced visible-light-driven photocatalytic activity toward dyes in water," Journal of Hazardous Materials, vol. 244-245, pp. 86-93, 2013.

[22] J. An, L. Zhu, N. Wang et al., "Photo-fenton like degradation of tetrabromobisphenol A with graphene $\mathrm{BiFeO}_{3}$ composite as a catalyst," Chemical Engineering Journal, vol. 219, pp. 225-237, 2013.

[23] Z. Li, Y. Shen, C. Yang et al., "Significant enhancement in the visible light photocatalytic properties of $\mathrm{BiFeO}_{3}$-graphene nanohybrids," Journal of Materials Chemistry A, vol. 1, pp. 823-829, 2013.

[24] J. Lin, C. Zhang, Z. Yan et al., "3-dimensional graphene carbon nanotube carpet-based microsupercapacitors with high electrochemical performance," Nano Letters, vol. 13, no. 1, pp. 72-78, 2013.

[25] Z. Yan, L. Ma, Y. Zhu et al., "Three-dimensional metal-graphene-nanotube multifunctional hybrid materials," ACS Nano, vol. 7, no. 1, pp. 58-64, 2013.

[26] Y. Liang, Y. Li, H. Wang et al., " $\mathrm{Co}_{3} \mathrm{O}_{4}$ nanocrystals on graphene as a synergistic catalyst for oxygen reduction reaction," Nature Materials, vol. 10, no. 10, pp. 780-786, 2011.

[27] T. Li, J. Shen, N. Li, and M. Ye, "Hydrothermal preparation, characterization and enhanced properties of reduced graphene$\mathrm{BiFeO}_{3}$ nanocomposite," Materials Letters, vol. 91, pp. 42-44, 2013.
[28] V. Singh, D. Joung, L. Zhai, S. Das, S. I. Khondaker, and S. Seal, "Graphene based materials: past, present and future," Progress in Materials Science, vol. 56, no. 8, pp. 1178-1271, 2011.

[29] T. Xian, H. Yang, X. Shen, J. L. Jiang, Z. Q. Wei, and W. J. Feng, "Preparation of high-quality $\mathrm{BiFeO}_{3}$ nanopowders via a polyacrylamide gel route," Journal of Alloys and Compounds, vol. 480, no. 2, pp. 889-892, 2009.

[30] S. R. Morrison, Electrochemistry at Semiconductor and Oxidized Metal Electrode, Plenum Press, New York, NY, USA, 1980.

[31] T. Xian, H. Yang, J. Dai, Z. Wei, J. Ma, and W. Feng, "Preparation and photocatalytic performance of nano-bismuth ferrite with tunable size," Chinese Journal of Catalysis, vol. 32, no. 4, pp. 618623,2011

[32] H. Hotop and W. C. Lineberger, "Binding energies in atomic negative ions," Journal of Physical and Chemial Reference Data, vol. 4, no. 3, pp. 539-576, 1975.

[33] T. Andersen, H. K. Haugen, and H. Hotop, "Binding energies in atomic negative ions: III," Journal of Physical and Chemical Reference Data, vol. 28, no. 6, pp. 1511-1533, 1999. 

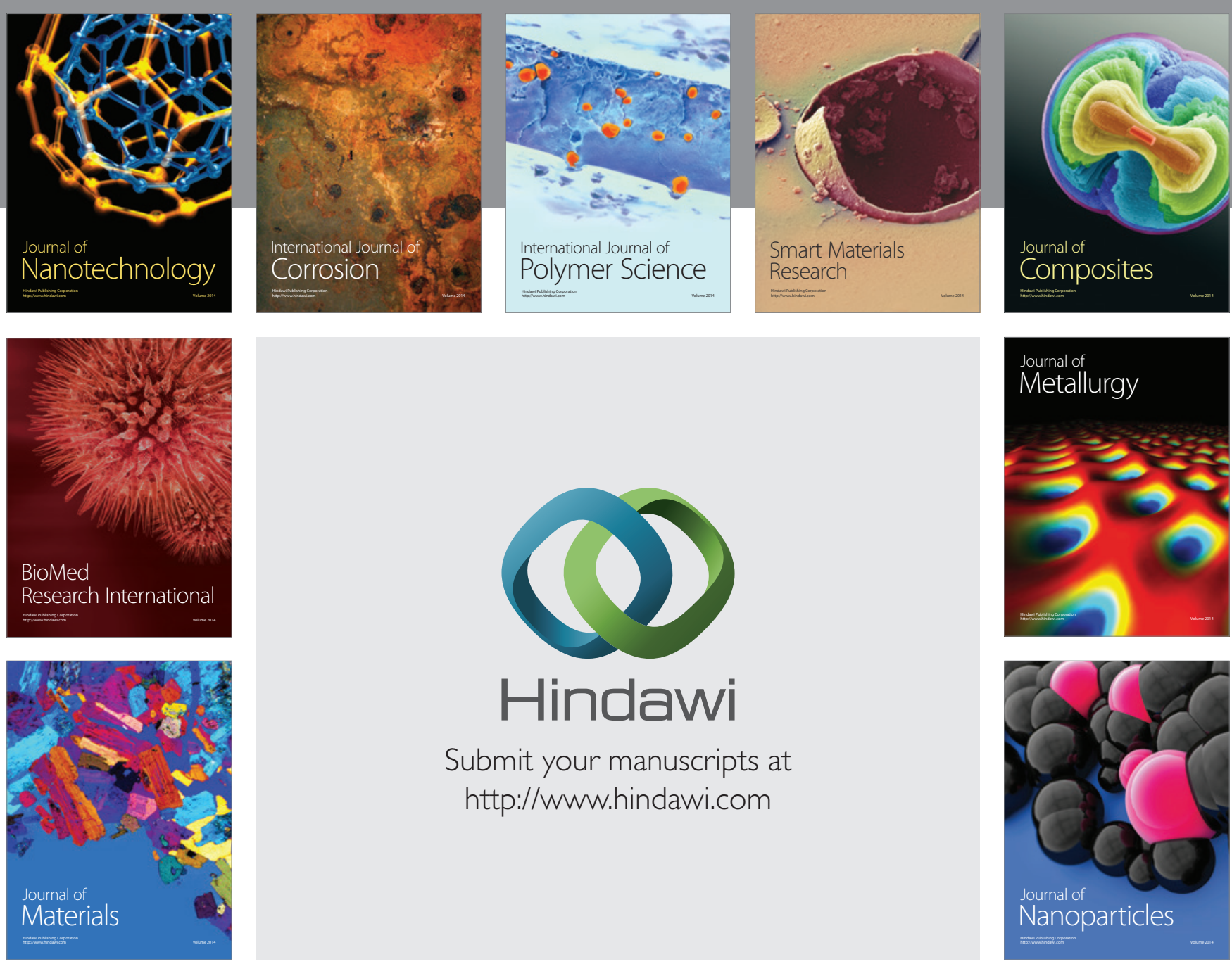

Submit your manuscripts at http://www.hindawi.com
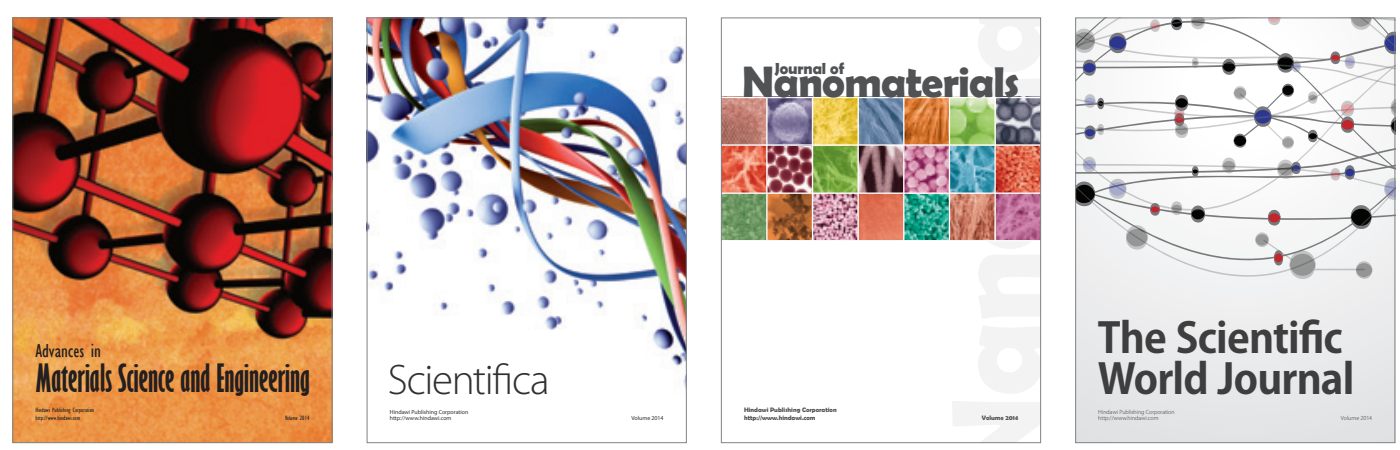

\section{The Scientific World Journal}
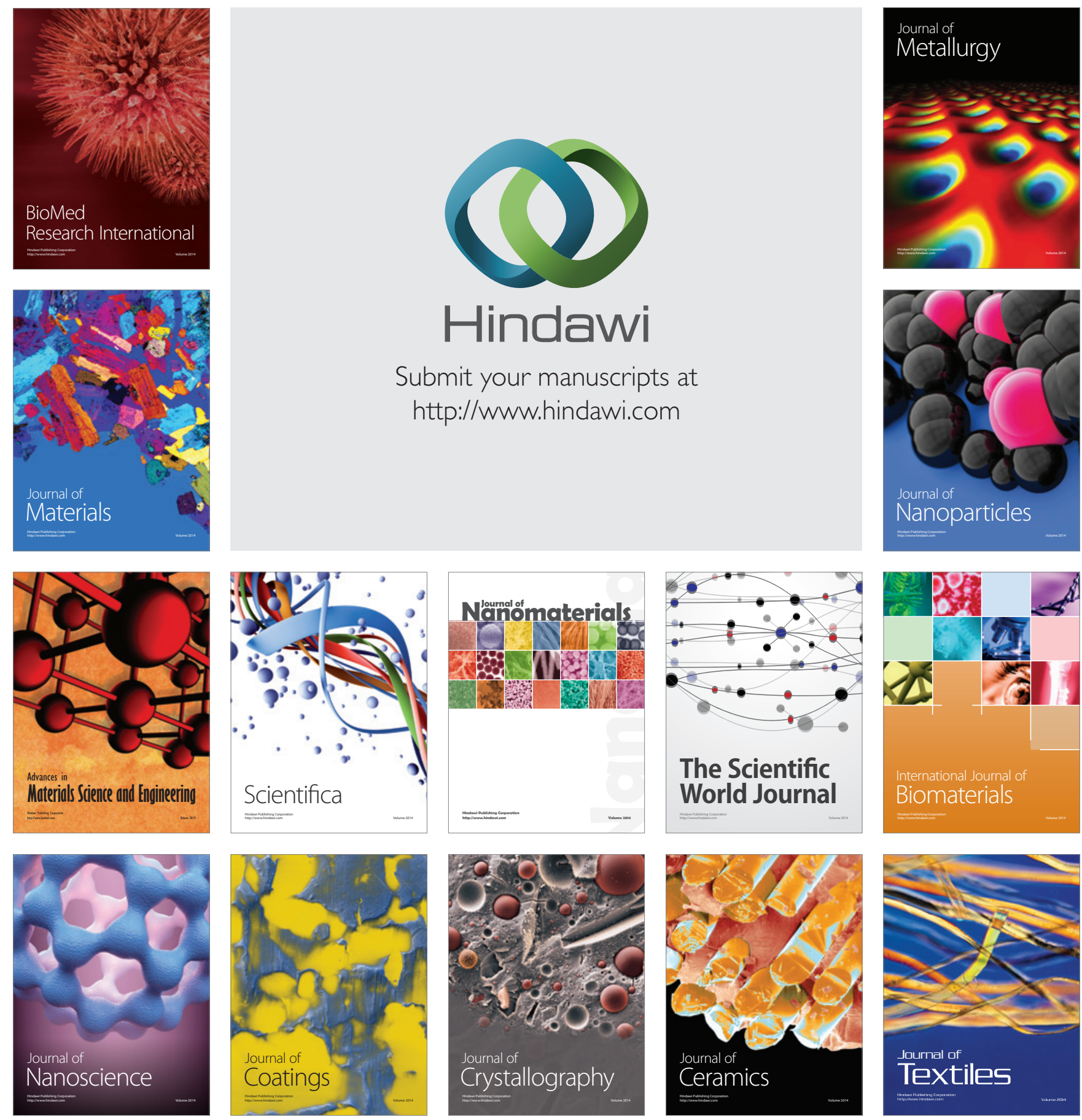